\title{
Visualization of return flow structure in mixed convection of gas over a heated circular plate in a horizontal flat duct
}

\author{
J.L. Tuh*, T.F. Lin \\ Department of Mechanical Engineering, National Chiao Tung University, Hsinchu 30010, Taiwan, ROC \\ Received 9 December 2002; accepted 14 April 2003 \\ Communicated by K. Nakajima
}

\begin{abstract}
Experimental flow visualization is conducted here to investigate the buoyancy-induced return flow structure in mixed convection of gas over a heated circular plate mounted on the bottom of a horizontal flat duct, simulating that in a horizontal longitudinal flow reactor. In particular, how the return flow induced in the upstream portion of the duct is related to the vortex flow in the downstream is delineated. The results show that at a low buoyancy-to-inertia ratio only steady longitudinal vortex rolls (L-rolls) are induced in the exit half of the duct and no return flow appears in the duct. At an intermediate buoyancy-to-inertia ratio a small flow recirculation exists around the duct inlet near the top wall and the L-rolls become unsteady. When the buoyancy-to-inertia ratio is high and the Reynolds number is small with $R e<10$, the upstream return flow is strong and occupies a large region. There is another return flow zone around the exit end of the duct. Over the heated circular plate the flow is dominated by the moving transverse rolls (T-rolls). Moreover, the return flow around the duct inlet at steady or statistically stable state is in the form of a semicircular roll around the upstream edge of the circular plate. Flow visualization also reveals that the splitting of the downstream tip of the return flow zone and the rising of the buoyancy-driven spanwisely extended thermal under the tip generate a pair of counter-rotating transverse rolls in the entry portion of the duct. In addition, the criterion for the onset of the return flow near the duct inlet, the size and the center position of the return flow, based on the present experimental data, are correlated empirically.
\end{abstract}

(C) 2003 Elsevier B.V. All rights reserved.

Keywords: A1. Convection; A1. Fluid flows; A3. Metalorganic chemical vapor deposition

\section{Introduction}

The presence of mixed convective secondary flow recirculations at supercritical buoyancy can result in a non-uniform vapor deposition on silicon wafers and is harmful to the thin-film properties. Especially, the existence of the return flow zone will blur the interfaces in the multi-layer

\footnotetext{
*Corresponding author.
}

thin-film structures and is most unwanted in the metal-organic chemical vapor deposition (MOCVD) processes [1]. The previous studies on the return flow mainly focused on the mixed convection in rectangular ducts of different aspect ratios heated uniformly from below and cooled uniformly from above. The return flow is therefore driven by the temperature differences between the horizontal top and bottom plates and between the cold inlet gas and hot bottom plate. In view of 


\begin{tabular}{|c|c|c|c|}
\hline \multicolumn{4}{|c|}{ Nomenclature } \\
\hline$A$ & aspect ratio, $b / d$ & $\Delta T$ & $T_{\mathrm{cp}}-T_{\mathrm{in}}$ \\
\hline$b, d$ & channel width, height & $T$ & time, $\mathrm{s}$ \\
\hline$G$ & gravitational acceleration & $t_{\mathrm{p}}$ & period of the flow oscillation, s \\
\hline$G r$ & Grashof number, $g \beta d^{3} \Delta T / \gamma^{2}$ & $W_{\mathrm{m}}$ & average velocity component in the $z$ \\
\hline$L$ & length of test section & & direction \\
\hline$l_{Y}, l_{Z}$ & the vertical and axial size of the return & $X, Y, Z$ & dimensional Cartesian coordinates \\
\hline & flow & $x, y, z$ & dimensionless Cartesian coordinates \\
\hline$R a$ & Rayleigh number, $\beta g d^{3} \Delta T / \alpha v$ & & scaled with $b, d, l$ \\
\hline & Reynolds number, $W_{\mathrm{m}} d / v$ & & \\
\hline$G r / R e^{2}$ & buoyancy-to-inertia ratio & \multicolumn{2}{|c|}{ Greek letters } \\
\hline & temperature & $\alpha$ & thermal diffusivity \\
\hline$T_{\mathrm{in}}, T_{\mathrm{cp}}$ & temperature at inlet of the test section & $\beta$ & thermal expansion coefficient \\
\hline & and the circular copper plates & $\gamma$ & kinematic viscosity \\
\hline
\end{tabular}

the fact that the buoyancy-driven secondary flow is very sensitive to the geometry of the system [2], the return flow characteristics predicted from these studies are expected to be somewhat different from those in the real MOCVD processes where the heated wafers are circular in shape. Particularly, the return flow will be highly three dimensional. In the present model experiment, we explore the characteristics of the return flow induced in the mixed convection of gas moving over a uniformly heated circular plate embedded in the bottom of a horizontal flat duct, simulating that encountered in horizontal longitudinal flow reactors. Besides, how the return flow is related to the vortex flow induced in the downstream region of the duct will be examined.

In the following the literature relevant to the present study is briefly reviewed. In the mixed convective flow through a flat duct with a uniformly heated bottom the return flow is known to result from the strong upward thermal buoyancy as the cold entering gas is suddenly heated in the entry heated section of the duct. The cold flow from the upstream is forced to lift up first and then bounces back to move upstream by the strong retarding force of the upward buoyancy, forming a reverse flow zone. At high buoyancy the return flow can penetrate significantly into the upstream unheated section of the duct and becomes highly elongated. When the main flow is at a high
Reynolds number, the return flow can extend substantially into the heated section of the duct and is also highly elongated.

The return flow encountered in the horizontal MOCVD reactors was first investigated by Eversteyn et al. [3] by visualizing the flow. They identified a particle-free zone above the susceptor and erroneously interpreted this zone as a stagnant layer of fluid. Instead, it is well known today as the return flow zone. Kamotani et al. [4] experimentally investigated the thermal instability of a laminar flow in a horizontal flat duct heated from below and found that at a high buoyancy-toinertia ratio with $G r / R e^{2} \gg 1$ a reverse flow zone was induced near the upper plate. Flow visualization conducted by Giling [5] to investigate flow pattern and temperature profile in horizontal MOCVD reactors proved that there was no stagnant layer above the susceptor. According to the experimental and numerical study of $\mathrm{N}_{2}$ and $\mathrm{H}_{2}$ gas flows in a bottom-heated quartz reactor with a rectangular cross section, Visser et al. [1] indicated that the returning flow was mainly dominated by two dimensionless parameters, the Grashof and Reynolds numbers, $G r$ and Re. They proposed a critical level $\alpha_{\text {crit }}$ for the mixed convection parameter $\mathrm{Gr} / \mathrm{Re}^{\kappa}$ and showed that no return flow occurred when $G r / R e^{\kappa}<\alpha_{\text {crit }}$. The exponent $\kappa$ is equal to 1 at low Reynolds numbers $(R e \leqslant 4)$ and goes to 2 at higher Reynolds numbers 
$(R e \geqslant 8)$. A similar study conducted by Fotiadis et al. [6] also indicated that either the heated susceptor was placed in the bottom or top wall, a flow recirculation could be induced. Moreover, the recirculation was noted in the upper portion of the reactor.

A two-dimensional numerical simulation carried out by Ouazzani et al. [7] to predict the buoyancydriven flow recirculations in the entrance region of a horizontal MOCVD reactor manifested that the presence of the return flow could result in an increase of film growth rate at the leading edge of the substrate and a decrease in the downstream region. Later, they [8] extended the analysis to include the three-dimensional effects and found that at a high $R a / R e$ ratio, a buoyancy-induced reversal flow existed in the transition region between the isothermal entrance and the reaction section. Einset et al. [9] moved further to define the onset of recirculation flows in the entrance region of horizontal CVD reactors based on the relative magnitudes of the vertical and horizontal pressure gradients in the flow. The pressure effects are independent of whether the heated substrate faces up or down, which explains their experimental observation that the flow recirculations appear at the same position in either configuration. Besides, they noted that the recirculation rolls located near the top wall of the reactor and rotated counter-clockwisely for both top- and bottomheated reactors. Ingle and Mountziaris [10] again used a two-dimensional numerical simulation to investigate the onset of transverse buoyancydriven circulations in a horizontal flat duct consisting of a cool upstream section, a middle section with a heated bottom wall, and a cool downstream section. Their results showed a transverse recirculation formed in the middle section near the top wall above the leading edge of the hot bottom wall, which rotated in a counterclockwise direction. The other one formed near the bottom wall above the exit end of the hot bottom plate and rotated in a clockwise direction. At increasing inlet velocity, the downstream transverse recirculation was eliminated first and the upstream one shrank significantly. In addition, they proposed two criteria for the absence of the transverse recirculation as $\left(G r / R e^{2}\right)<100$ for
$10^{-3}<\operatorname{Re} \leqslant 4$, and $\left(G r / R e^{2}\right)<25$ for $4 \leqslant R e<100$. A similar two-dimensional numerical simulation from Ingham et al. [11,12] predicted that the transverse flow recirculation could extend to the upstream of the wall temperature discontinuity. The onset of the return flow for the heated lower wall was shown to occur at $G r / R e^{2} \simeq 17$ for $R e=$ 10. This critical value of $G r / R e^{2}$ slowly decreased at increasing $R e$. Besides, the recirculation zone is larger for a lower $R e$. Makhviladze and Martjushenko [13] conducted two- and three-dimensional numerical simulations to study the return flow in horizontal bottom-wall-heated CVD reactors. They showed the formation mechanism of the return flow and the return flow could be suppressed by cooling the side walls and/or by reducing the width of the reactors. The characteristics of three-dimensional flow, heat and mass transfer in a horizontal CVD reactor were numerically investigated by Park and Pak [14]. They concluded that for a large $\mathrm{Gr} / \mathrm{Re}^{2}$ the return flow occurred at the leading edge of the susceptor, and it caused an increase in the growth rate of the film at this region and a decrease in the downstream of the susceptor.

The above literature review clearly indicates that the previous studies of the buoyancy-driven return flow mainly focus on mixed convection of gas in horizontal rectangular ducts uniformly heated from below and cooled from above. No study has been conducted to explore how the shape of the heated bottom plate affects the return flow. In the present study flow visualization is carried out to unravel the characteristics of the return flow induced in a mixed convective gas flow over a circular isothermally heated plate embedded in the bottom of a horizontal flat duct. The top plate of the duct is thermally insulated. The influences of the Reynolds and Rayleigh numbers will be examined in detail.

\section{Experimental apparatus and procedures}

\subsection{Experimental apparatus}

Fig. 1 shows a schematic view of the present experimental apparatus designed to study the 

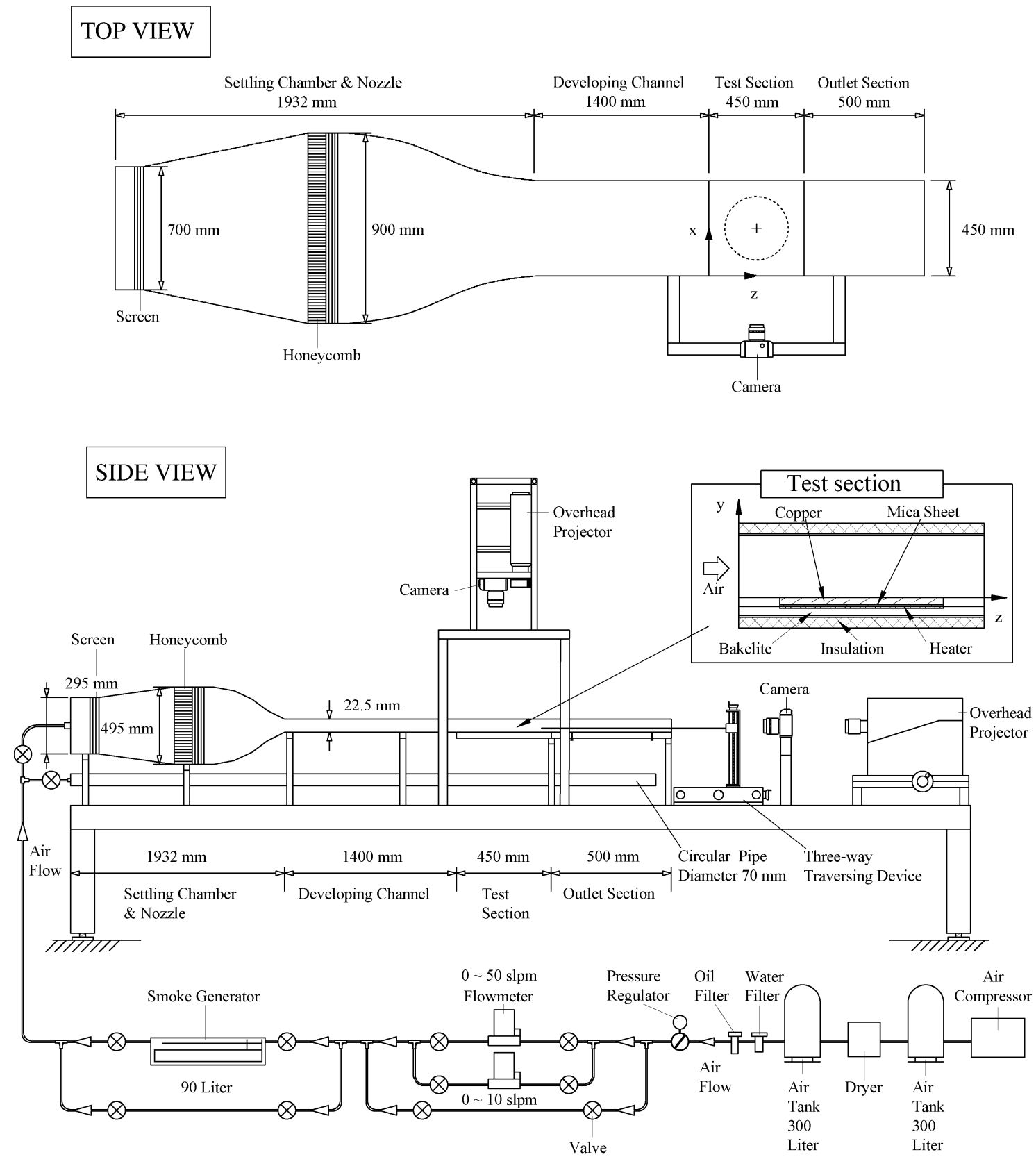

Fig. 1. Schematic of experimental apparatus and the chosen coordinate system for the test section.

buoyancy-induced return flow structures in the mixed convective gas flow over a heated circular plate embedded in the bottom of a horizontal plane channel (a high aspect ratio rectangular duct) and the adopted coordinate system. To reduce the cost of the experiment, we use air as the working fluid to replace the inert gases normally employed in real MOCVD processes. In view of the similar thermodynamic and thermophysical properties for various gases, the 
results obtained here are still applicable to the MOCVD systems. The test section has a cross section of $22.5 \mathrm{~mm}$ in height and $450 \mathrm{~mm}$ in width, providing an aspect ratio of $A=20$, and has a total length of $450 \mathrm{~mm}$. The side and top walls of the duct are constructed of 10-mm-thick transparent acrylic plates to allow the visualization of vortex flow patterns. Moreover, the outside surfaces of the top and side walls are thermally insulated by a superlon insulator of $13 \mathrm{~mm}$ thickness. During the flow visualization the insulator can be opened temporally. The bottom of the test section is a thick flat bakelite plate embedded with a 15 -mm-thick, high-purity circular copper plate of $300 \mathrm{~mm}$ diameter to model a 12in semiconductor substrate. The upper surfaces of the bakelite and copper plates are kept at the same horizontal level so that the air flow does not experience any step when moving over the copper plate. To obtain the uniform plate temperature, the heating elements attached onto the lower surface of the copper plate are divided concentrically into seven zones and the heater for each zone is independently controlled by a GW GPC 3030D laboratory power supply. Besides, a mica sheet is placed between the copper plate and heating elements to prevent the electric current leaking to the copper plate.

A good control of the flow condition upstream of the test section is essential in the experiment. More specifically, at the inlet of the loop, the working fluid (air) is driven by a $7.5 \mathrm{hp}$ air compressor and sent through a dryer installed with water vapor and oil filters. This dry air then moves into the high-pressure tank. To proceed with the experiment, the airflow is further controlled by a pressure regulator and its volume flow rate is measured by Brooks $5850 \mathrm{E}$ and/or $5851 \mathrm{E}$ flow controllers, both with an accuracy of $1 \%$. These two flow controllers individually operate in the ranges of $0-10$ and $0-501 / \mathrm{min}$. Through a flexible tube, the air enters the settling chamber, in which four fine-mesh screens, a divergent buffer section, a honeycomb and another four fine-mesh screens are installed in sequence to reduce the turbulence in the air flow. The air turbulence is further suppressed by passing the air through a contraction nozzle with a contraction ratio of $44: 1$, which provides a nearly uniform velocity at the inlet of the developing section.

The developing section is $1400 \mathrm{~mm}$ in length, approximately 62 times of the duct height. This insures the flow to be fully developed at the inlet of the test section for $R e \leqslant 100$. An insulated outlet section of $450 \mathrm{~mm}$ long is added to the test section to reduce the effects of the disturbances from discharging the flow to the ambient. The developing section and outlet sections are both made of 10 -mm-thick acrylic plate, whereas the settling chamber and contraction nozzle are made of stainless-steel plates. The settling chamber, developing section, test section and outlet section are all thermally insulated with a 20 -mm-thick Superlon insulator and the entire loop is fixed on a rigid supporting frame.

Visualization of the buoyancy-driven vortex flow in the test section is realized by injecting smoke at some distance ahead of the settling chamber. The smoke is produced by a smoke generator, which is a cubic space with the incense burned in it. By keeping the smoke concentration at a suitable level, the incense particles can be illuminated by a plane light sheet from a $550-\mathrm{W}$ overhead projector. With an adjustable knife edge a sharp contrast could be achieved between the duct walls and smoke. The flow photos from the top, side and end views of the test section can then be taken. The exposure time is about $1 / 125 \mathrm{~s}$ in taking the photos.

The temperature of the heated copper plate is measured by 17 calibrated and electrically insulated T-type thermocouples embedded at selected locations in the plate. The thermocouple beads are fixed at about $1 \mathrm{~mm}$ from the upper surface of the copper plate through the small holes drilled from the backside of the plate. A T-type thermocouple is also used to measure the inlet air temperature at locations just upstream of the test section. The signals from the thermocouples are recorded by the Hewlett-Packard 3852A data acquisition system with a resolution of $\pm 0.05^{\circ} \mathrm{C}$. It was noted that in all tests the maximum temperature differences between any two locations in the copper plate were below $0.1^{\circ} \mathrm{C}$. The error in the temperature difference between the copper surface and the air at the duct inlet is estimated to be within $\pm 0.1^{\circ} \mathrm{C}$. 


\subsection{Preliminary investigations of flow field}

In order to confirm the fully developed condition at the entrance of the test section, measurements using a hot-wire anemometer (DANTEC probe Type 55P01 with 56C17 CTA bridge) in connection with a HP data acquisition system (the Hewlett-Packard VXI series-E1411B multimeter and E-1347A multiplexers) for the inlet velocity profiles are conducted. For calibrating the hot wire, the pipe-flow method that the probe is placed in the center of a fully developed laminar pipe flow is used. The total volume flow rate is estimated and the pipe center velocity is calculated from the parabolic distribution. The measured data are in good agreement with the analytical results given by Shah and London [15]. Besides, the turbulence level of the inlet stream is all within $1 \%$. This implies that the effects of the free stream turbulence on the mixed convective flow characteristics are moderate.

\subsection{Experimental procedures}

For convenience, experiments are designated according to the Reynolds and Rayleigh numbers based on the inlet conditions and the mean air speed in the test section calculated from the total flow rate. In each test the flow controller is first set at the predetermined level to impose a desired, fully developed flow through the entire test section. The power supplies are then turned on to raise the copper plate temperature. Usually, it takes about $3 \mathrm{~h}$ for the Rayleigh number to be raised to the test point and another $2 \mathrm{~h}$ are needed to maintain the vortex flow at steady or statistically stable state. After the steady or statistically stable state is reached, we start the flow visualization.

\subsection{Uncertainty analysis}

Uncertainties in the Rayleigh number $R a$, Reynolds number $R e$ and other independent parameters are estimated in the light of the standard procedures proposed by Kline and McClintock [16]. The uncertainties of the thermophysical properties of air are included in the analysis. The fundamental thermophysical properties of the working fluid (air) are $\alpha=0.22\left(\mathrm{~cm}^{2} / \mathrm{s}\right)$, $\beta=0.0034(1 / \mathrm{K})$ and $v=0.162\left(\mathrm{~cm}^{2} / \mathrm{s}\right)$ at $30^{\circ} \mathrm{C}$ and 0.997 bar. The fluid properties are further corrected based on the temperature and pressure detected at the inlet of the test section. In addition, the deviation of the temperature among the detecting points in the circular copper plate and the control unsteadiness are also accounted for in the evaluation of the data uncertainties. The analysis shows that the uncertainties of temperature, volume flow rate, dimensions, Reynolds number and Rayleigh number measurements are estimated to be less than $\pm 1.5^{\circ} \mathrm{C}, \pm 1 \%$, $\pm 0.05 \mathrm{~mm}, \pm 3 \%$ and $\pm 5 \%$, respectively.

\section{Results and discussion}

Selected results from the present study are presented in the following to illustrate various aspects of the return flow induced in a low Reynolds number mixed convective gas flow over a circular heated plate in the horizontal flat duct. Attention will be focused on how the geometry of this circular heated bottom plate affects the return flow characteristics. The influences of the Reynolds and Rayleigh numbers on the return flow will be examined in detail.

\subsection{Return flow at steady or statistically stable state}

To reveal the effects of the buoyancy-to-inertia ratio $G r / R e^{2}$ on the buoyancy-driven return flow, the side view flow photos taken at the midspan of the duct $(x=0.5)$ for selected cases at steady or statistically stable state with the Rayleigh number $R a$ fixed at 11,754 for decreasing Reynolds numbers are presented in Fig. 2. The results indicate that at the low buoyancy-to-inertia ratios for $R e \geqslant 28.1$ the flow streams in the entry half of the duct are deflected by the upward buoyancy to a certain degree. The forced main flow coming from the upstream is not reversed and no return flow zone exists in the midspan of the duct $(x=0.5)$ according to the side view flow photos shown in Figs. 2(a)-(d). It is of interest to note that at 


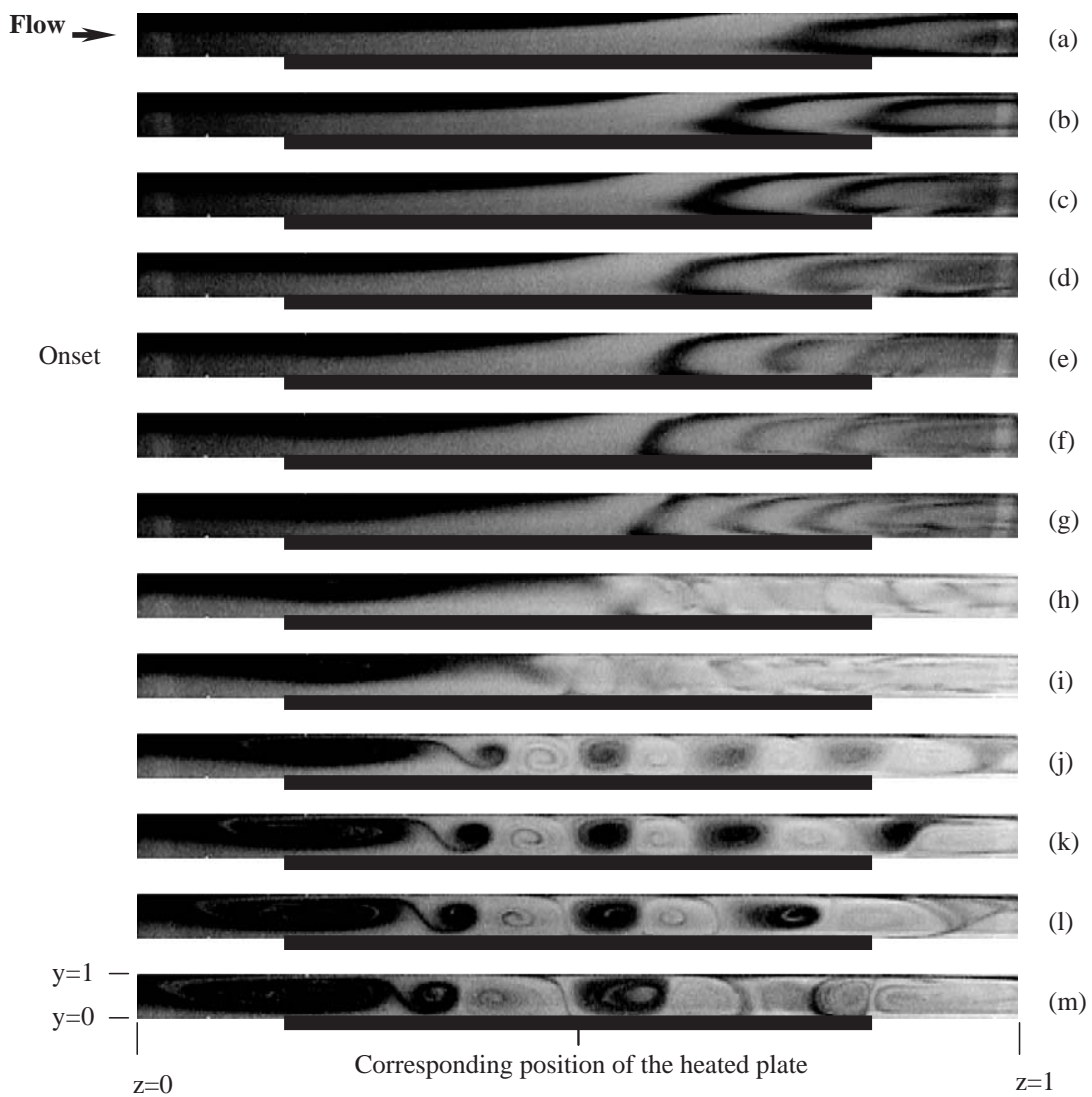

Fig. 2. Side view flow photos at steady or statistical state for $R a=11,754$ on the plane $x=0.5$ for (a) $R e=42.1$, (b) $R e=35.1$, (c) $R e=30.4$, (d) $R e=28.1$, (e) $R e=22.8$, (f) $R e=21.1$, (g) $R e=18.7$, (h) $R e=16.4$, (i) $R e=14.0$, (j) $R e=11.7$, (k) $R e=9.4$, (1) $R e=7.8$, and (m) $R e=4.7$.

$R e=30.4$ some longitudinal recirculations in the form of parallel vortex rolls with all their axes in the forced flow direction are induced in the exit half of the duct, as evident from the steady top and side view flow photos shown in Fig. 3 for $R e=$ 30.4 and $R a=11,754$. The characteristics of the longitudinal vortex rolls and their generation have been examined in a recent study of Tuh and Lin [17]. These top and side view flow photos manifest that the return flow does not exist in the entire duct. Now as the Reynolds number is lowered to 22.8 , the forced main flow is reversed by the upward buoyancy at this higher $G r / R e^{2}$ and a very small return flow zone is seen near the upper wall in the entry portion of the duct (Fig. 2(e)). In the exit half of the duct the flow is also dominated by the longitudinal vortex rolls. Note that the return flow is stronger and occupies a larger region when $R e$ is lowered further, as evident from the results in Figs. 2(f)-(m). It should be mentioned that at $R e=18.7$ the longitudinal rolls in the downstream are somewhat unsteady, because of the higher buoyancy-to-inertia ratio associated with the decreasing inertia force of the flow. For the lower $R e$ of 16.4 and 14.0 irregular vortex rolls prevail in the exit half of the duct (Figs. 2(h) and (i)). At even lower $R e$ of $11.7,9.4,7.8$ and 4.7 transverse recirculations with all their axes normal to the forced flow direction appear in the duct (Figs. 2(j)(m)). It should be mentioned that the transverse recirculations are rolling forward at a constant speed. Note that at the lowest $R e$ of 4.7 the return flow is rather strong and nearly dominates the entire test section (Fig. 2(m)). The return flow 


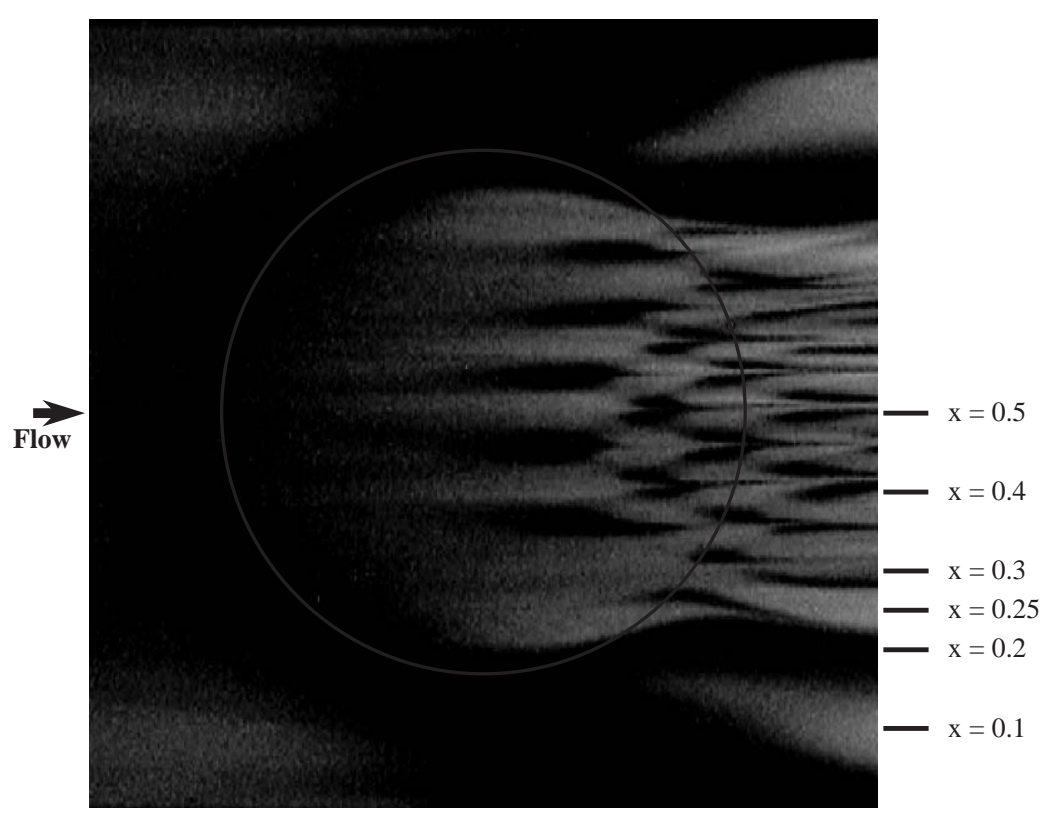

(a)

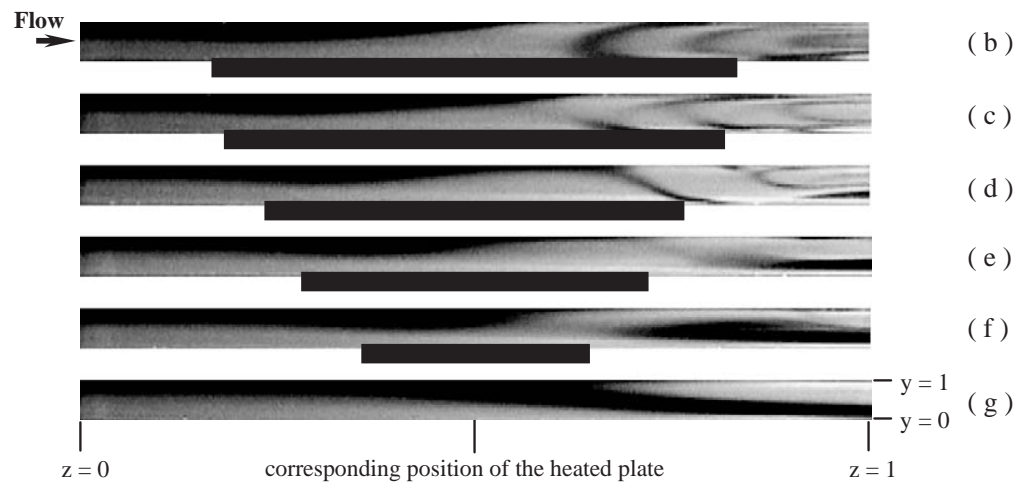

Fig. 3. Top view flow photo at $y=0.5$ (a) and side view flow photos on the planes: (b) $x=0.5$, (c) $x=0.4$, (d) $x=0.3$, (e) $x=0.25$, (f) $x=0.2$, and (g) $x=0.1$ at steady state for $R e=30.4$ and $R a=11,754$.

structure combined with transverse vortices can be clearly illustrated by the top and side view flow photos shown in Fig. 4 for $R e=10.1$ and $R a=$ 11,594. Note that the moving transverse rolls dominate in the region right above the circular heated plate and the upstream return flow is in the form of a semicircular roll surrounding the upstream edge of the copper plate. The return flow in the downstream region of the duct deforms to a certain degree. It is worth noting that the return flow in this unique form of a semicircular roll driven by the circular heated plate is very different from that driven by the uniformly heated rectangular bottom plate. In that case the return flow is nearly two dimensional.

The effects of the buoyancy-to-inertia ratio on the return flow are further illustrated by examining the long time side flow photos taken at $x=0.5$, shown in Fig. 5, at increasing Rayleigh numbers for the Reynolds number fixed at a very low level of 7.8. The results again indicate that at a higher buoyancy-to-inertia ratio for a higher Rayleigh number a stronger return flow is induced and the return flow zones appearing in the duct inlet and 


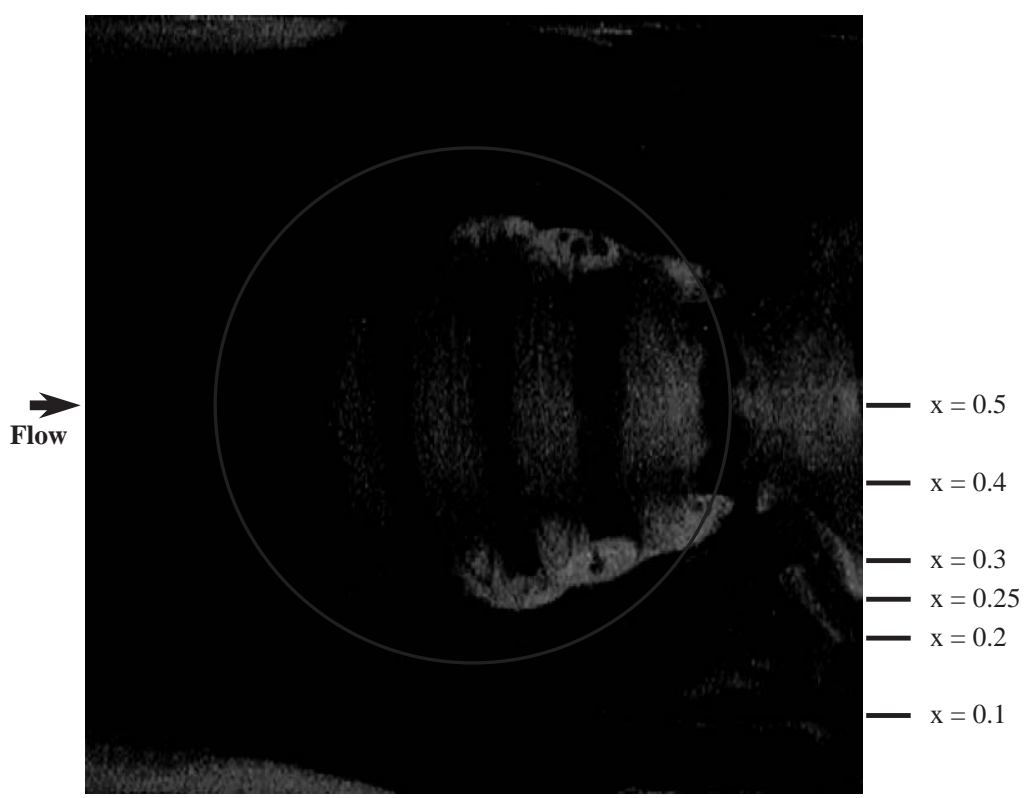

( a )

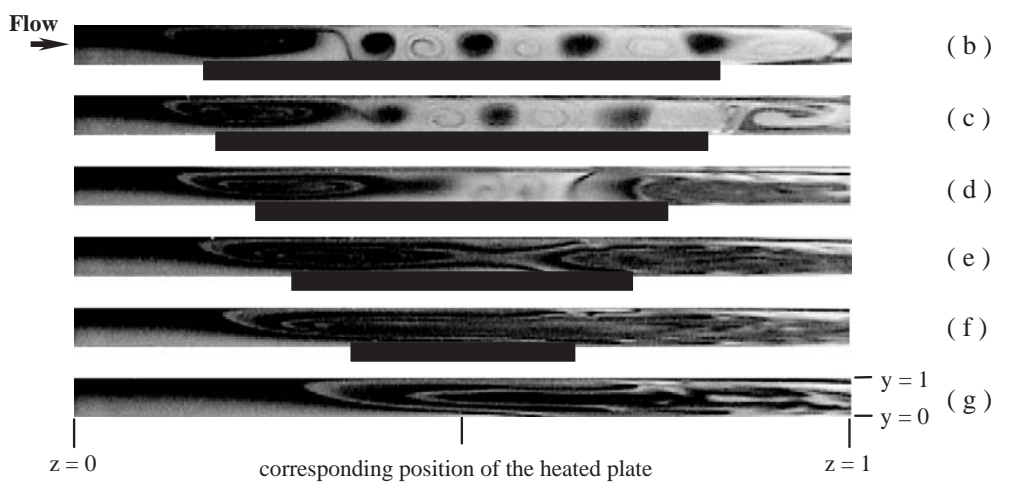

Fig. 4. Top view flow photo at $y=0.5$ (a) and side view flow photos on the planes: (b) $x=0.5$, (c) $x=0.4$, (d) $x=0.3$, (e) $x=0.25$, (f) $x=0.2$, and $(\mathrm{g}) x=0.1$ at statistical state for $R e=10.1$ and $R a=11,594$.

exit are larger. A close inspection of the photos reveals that for these cases the reverse flow almost dominates the entire entry portion of the duct and the main flow is forced to move at a relatively higher speed in the narrow space under the return flow zone to enter the duct. This much faster forced flow near the heated plate under the return flow zone explains the higher film growth rate at the leading edge of the substrate [7,14]. Moreover, at this low Reynolds number transverse vortex rolls appear in the immediate downstream of the return flow zone. This leads us to examine the possible close relationship between the return flow and transverse rolls. This is carried out by visualizing the time evolution of the return flow. The results are shown in Fig. 6 by presenting the side view flow photos at the midspan of the duct at selected instants of time for a typical case with $R e=10.1$ and $R a=11,594$. The results manifest that the transverse roll pair closest to the duct inlet is generated from the gradual splitting of the downstream tip of the return flow zone and the buoyancy-induced thermal right below the tip. The splitting of the tip of the return flow zone and the growth of the thermal can be clearly seen from the photos in Fig. 6. Having generated in the entry 


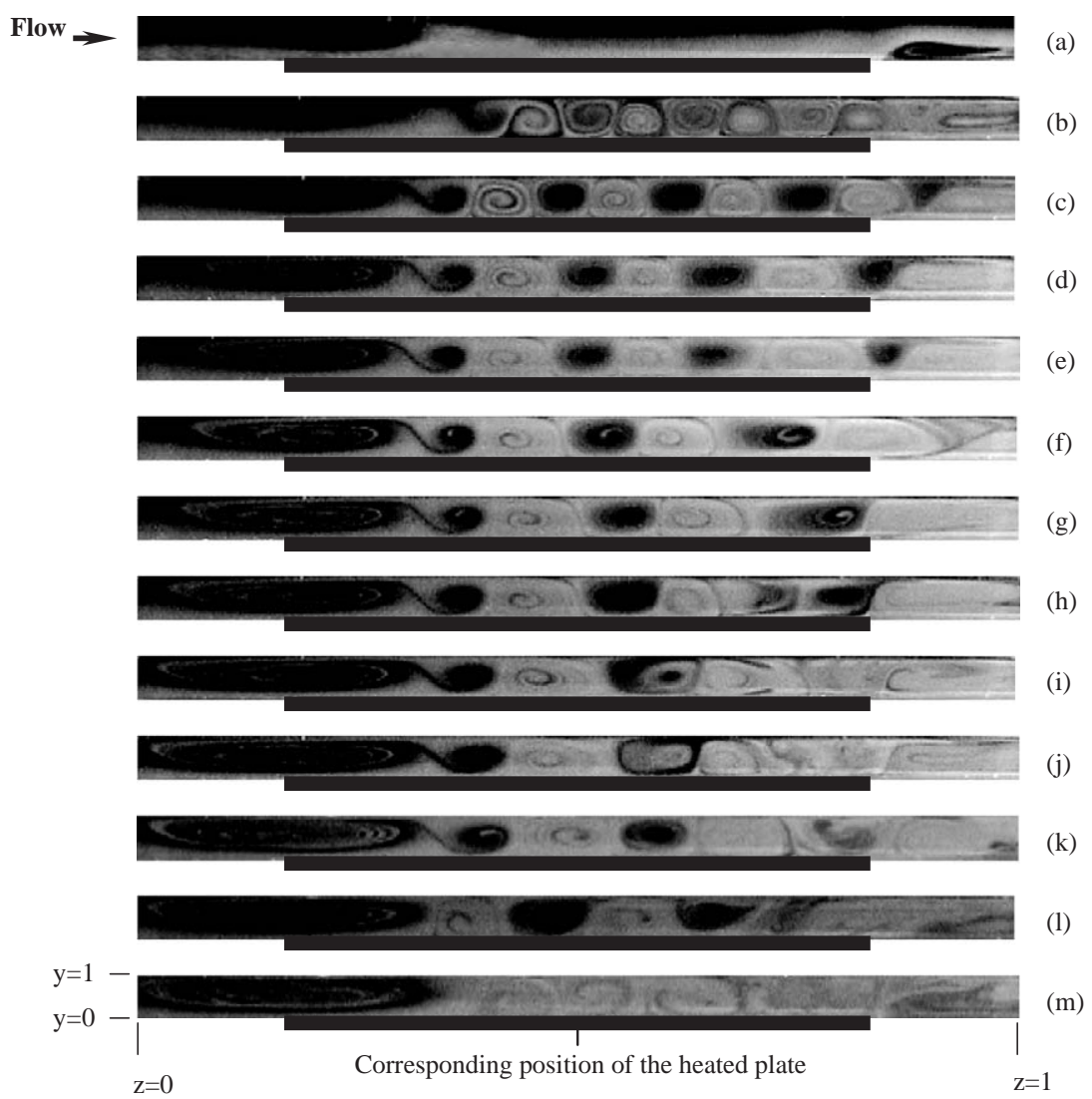

Fig. 5. Side view flow photos at statistical state for $R e=7.8$ on the plane $x=0.5$ for (a) $R a=3,196$, (b) $R a=5,236$, (c) $R a=6,477$, (d) $R a=9,465$, (e) $R a=10,703$, (f) $R a=11,754$, (g) $R a=14,198$, (h) $R a=15,088$, (i) $R a=17,005$, (j) $R a=19,598$, (k) $R a=$ 23,670, (l) $R a=25,688$, and (m) $R a=28,583$.

portion of the duct, the transverse rolls are pushed by the main flow to slowly move downstream. Note that during the downstream moving the rolls grow slightly in size. It is also noted that the downstream return flow zone including its shape, size and location is significantly affected by the downstream convection of the transverse rolls. The detailed characteristics of the transverse vortex flow driven by the present circular heated plate will be examined in near future.

\subsection{Criterion and some quantitative characteristics of return flow}

A very important task in the present study is to identify the critical condition for the onset of the buoyancy-induced return flow in mixed convection of air for this unique geometry of the circular heated surface. By inspecting the side view and top view flow photos for various Reynolds and Rayleigh numbers, the critical $R e$ and $R a$ of the return flow are plotted in Fig. 7. The results indicate that the critical buoyancy-to-inertia ratio $G r / R e^{2}$ decreases with the increase in the Reynolds number. According to the present data, the onset of the return flow driven by the circular heated plate placed in the bottom of a flat duct can be correlated as

$\frac{G r}{R e^{2}}=26+2860 R e^{-2}$

for $5 \leqslant R e \leqslant 40$ and $3200 \leqslant R a \leqslant 31,000$. 


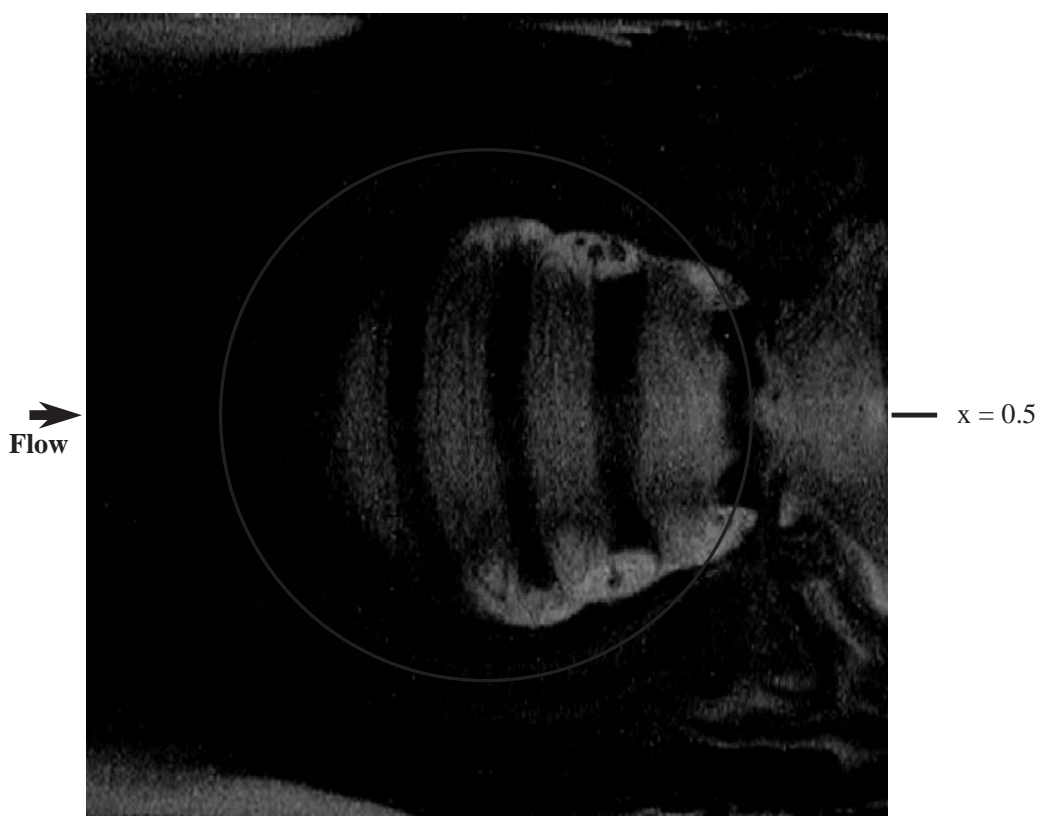

( a )

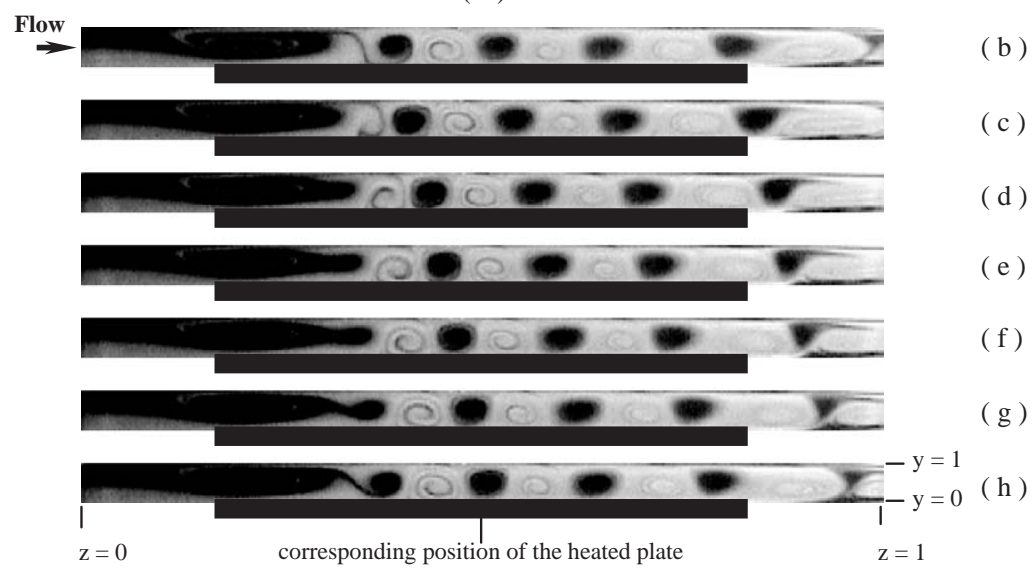

Fig. 6. Top view flow photo at $y=0.5$ (a) and side view flow photos for the central vertical plane at $x=0.5$ in a typical periodic cycle for $R e=10.1$ and $R a=11,594$ at time (b) $t$, (c) $t+1 / 7 t_{\mathrm{p}}$, (d) $t+2 / 7 t_{\mathrm{p}}$, (e) $t+3 / 7 t_{\mathrm{p}}$, (f) $t+4 / 7 t_{\mathrm{p}},(\mathrm{g}) t+5 / 7 t_{\mathrm{p}}$, and (h) $t+6 / 7 t_{\mathrm{p}}$ $\left(t_{\mathrm{p}}=7.14 \mathrm{~s}\right)$

Some quantitative characteristics of the return flow such as the size and location of the upstream return flow zone are inspected. More specifically, the streamwise and vertical extents and the position of the center of the upstream return flow zone at the midspan of the duct $(x=0.5)$ are measured from the side view flow photos, as illustrated in Fig. 8. The measured data for various
$R e$ and $R a$ are correlated empirically. The axial extent of the return flow $l_{Z}$ from this data correlation can be expressed as

$\frac{l_{Z}}{d}=4.1-1.38 R e^{0.5}+0.036 R a^{0.5}$.

The standard deviation of the above correlation is $10 \%$. The vertical extent of the return flow $l_{Y}$ from 
our data is correlated as

$\frac{l_{Y}}{d}=1.29-0.116 R e^{0.5}-20.9 R a^{-0.5}$.

The standard deviation of the above correlation is less than $8 \%$. With the origin chosen below the upstream tip of the return flow zone on the bottom plate, our data correlation for the center location of the return flow can be expressed as

$z_{\mathrm{c}}=0.11+0.037 R e^{0.5}-1.7 \times 10^{-6} R a$,

$y_{\mathrm{c}}=0.553+0.062 R e^{0.5}-0.001 R a^{0.5}$.

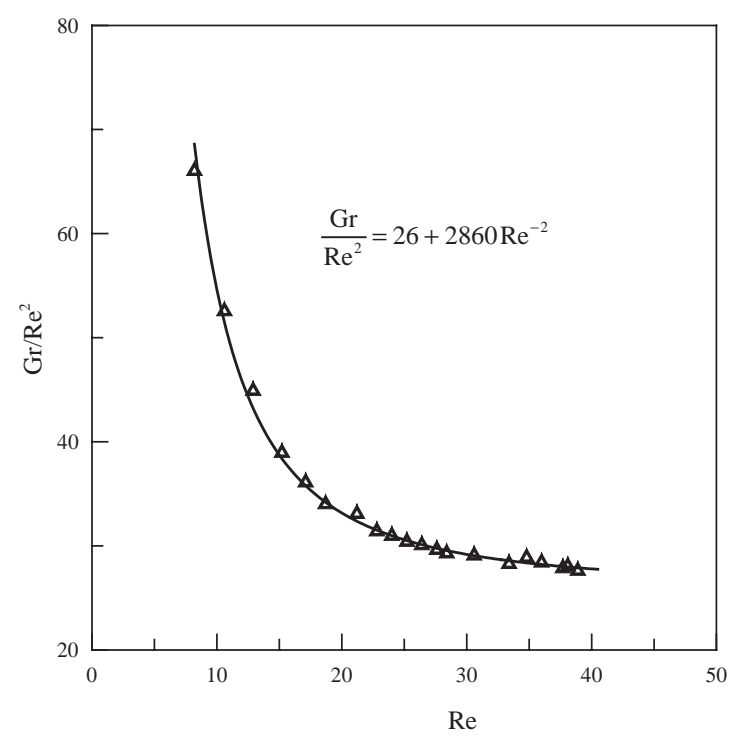

Fig. 7. Critical condition for the onset of return flow.
The standard deviations of the above two correlations are $6 \%$ and $8 \%$, respectively.

The above correlations quantitatively delineate the size growth of the return flow zone with the increases in the Reynolds and Rayleigh numbers. Note that the center of the return flow zone moves slightly upstream with increasing $R a$ at fixed $R e$ as shown in Fig. 5. Moreover, the center position of the return flow moves upstream with decreasing $R e$ at fixed $R a$ as shown in Fig. 2. Meanwhile, the upstream return flow grows in size at decreasing $R e$.

\section{Concluding remark}

An experiment with direct flow visualization has been carried out to investigate the buoyancydriven return flow in mixed convection gas flow over a horizontal substrate placed on the bottom of a horizontal flat duct. The influences of the Reynolds and Rayleigh numbers on the return flow have been examined. The major results obtained can be briefly summarized here. At a low buoyancy-to-inertia ratio, only steady longitudinal rolls with all their axes parallel with the duct axis prevail in the duct, and no reverse flow is observed in the duct. When we increase the buoyancy-to inertia ratio to an intermediate level the longitudinal rolls are induced at more upstream locations and become unsteady. Besides, the main flow starts to reverse and move upstream along the top wall of the duct and a small counterclockwise flow recirculation appears near the leading edge of the top wall. At a high buoyancy-to-inertia

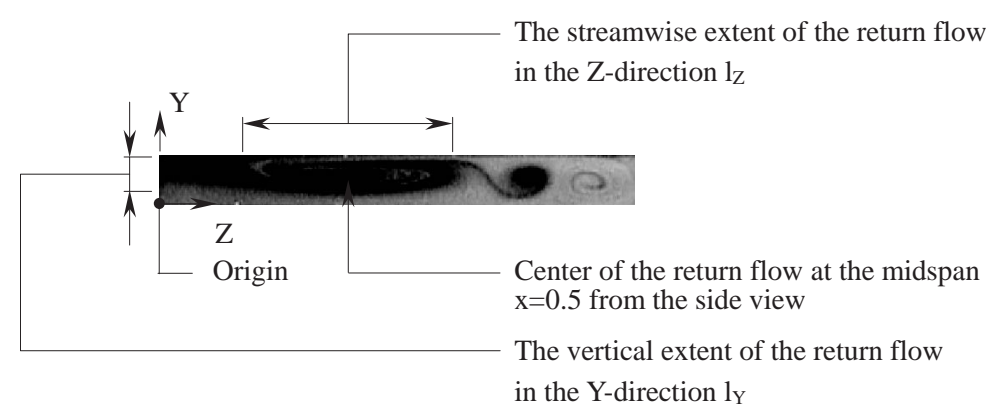

Fig. 8. Side view flow photo at $x=0.5$ indicating the size and location of the upstream return flow zone. 
ratio for a low Reynolds number, a returning flow zone is formed in the entry portion of the duct and transverse rolls prevail over the horizontal heated circular plate. The return flow is in the form of a semicircular roll around the upstream edge of the heated substrate. In addition, a downstream return flow zone is also induced near the exit end of the duct. The upstream return flow zone almost blocks the entire duct inlet. Moreover, the return flow grows in size and the center position of the upstream return flow zone migrates slightly towards the upstream and the top wall at increasing buoyancy-to-inertia ratio. To provide the quantitative reverse flow characteristics, the present data for the size and center position of the upstream return flow zone at the mid-span of the duct are correlated empirically. Besides, the criterion for the onset of the return flow is also proposed.

\section{Acknowledgements}

The financial support of this study by the engineering division of National Science Council of Taiwan, ROC through the Contract NSC 892212-E009-074 is greatly appreciated.

\section{References}

[1] E.P. Visser, C.R. Kleijn, C.A.M. Govers, C.J. Hoogendoorn, L.J. Giling, J. Crystal Growth 94 (1989) 929.

[2] E.L. Koschmieder, S.G. Pallas, Int. J. Heat Mass Transfer 17 (1974) 991.

[3] F.C. Eversteyn, P.J. Severin, C.H.J. van den Brekel, H.L. Peek, J. Electrochem. Soc. 117 (1970) 925.

[4] Y. Kamotani, S. Ostrach, H. Miao, ASME J. Heat Transfer 101 (1979) 222.

[5] L.J. Giling, J. Electrochem. Soc. 129 (1982) 634.

[6] D.I. Fotiadis, M. Boekholt, K.F. Jensen, W. Richter, J. Crystal Growth 100 (1990) 577.

[7] J. Ouazzani, K.C. Chiu, F. Rosenberger, J. Crystal Growth 91 (1988) 497.

[8] J. Ouazzani, F. Rosenberger, J. Crystal Growth 100 (1990) 545.

[9] E.O. Einset, K.F. Jensen, C.R. Kleijn, J. Crystal Growth 132 (1993) 483.

[10] N.K. Ingle, T.J. Mountziaris, J. Fluid Mech. 277 (1994) 249.

[11] D.B. Ingam, P. Watson, P.J. Heggs, Int. J. Heat Fluid Flow 16 (1995) 202.

[12] D.B. Ingham, P. Watson, P.J. Heggs, Int. J. Heat Mass Transfer 39 (1996) 437.

[13] T.M. Makhviladze, A.V. Martjushenko, Int. J. Heat Mass Transfer 16 (1998) 2529.

[14] K.W. Park, H.Y. Pak, Numer. Heat Transfer 37 (2000) 407.

[15] R.K. Shah, A.L. London, Laminar Flow Forced Convection in Ducts, Academic Press, New York, 1987, p. 196.

[16] S.J. Kline, F.A. McClintock, Mech. Eng. 75 (1953) 3.

[17] J.L. Tuh, T.F. Lin, Int. J. Heat Mass Transfer 46 (2003) 1341. 